\title{
Caracterización etnobotánica de los productos forestales no maderables (PFNM) en el corregimiento de Doña Josefa, Chocó, Colombia
}

\author{
Ethnobotany characterization of non-timber forest products \\ (NTFP) in the community of Doña Josefa, Chocó, Colombia
}

\author{
Angélica María Cogollo-Calderón ${ }^{1}$, Fabio García-Cossio ${ }^{2}$
}

\begin{abstract}
Resumen
En este estudio se identificaron 221 especies vegetales empleadas en diversos fines por los pobladores del corregimiento Doña Josefa, comunidad ubicada en el municipio del Atrato, departamento del Chocó, Colombia. Se evaluaron nueve categorías de uso medicinal, alimenticia, artesanal, ornamental, tóxica, mágico-religiosa, combustible, colorantes y plantas productoras de látex o exudados. La información se recopiló con base en el conocimiento tradicional que los habitantes tienen de estas plantas. Para ello se realizaron entrevistas semiestructuradas principalmente a las amas de casa, mineros y agricultores y luego se realizaron salidas de campo con pobladores de alto grado de comprensión en el tema, donde se recolectaron los ejemplares botánicos que luego fueron determinados taxonómicamente hasta la menor categoría posible, para depositarlos en el Herbario [CHOCO]. Las categorías más representativas en cuanto a número de especies fueron medicinal $(50,6 \%)$, alimenticia $(22,6 \%)$, combustible $(13,1 \%)$ y artesanal $(10,4 \%)$ destacándose las familias Malvaceae, Arecaceae, Asteraceae, Lamiaceae, Rubiaceae, Fabaceae/Mimosoideae, Gesneriaceae, Sapotaceae, Moraceae y Piperaceae. Con esto se evidencia la importancia que los productos forestales no maderables (PFNM) tienen para la comunidad de Doña Josefa, en donde son fuente principalmente de alimentos, medicinas, artesanías y de otros productos con grandes posibilidades de desarrollo económico, si se manejan apuntando hacia la gestión sostenible de los recursos naturales, la conservación de la biodiversidad y los servicios ecosistémicos que nos brindan.
\end{abstract}

Palabras clave: PFNM; Categorías de uso; Comunidades del Atrato; Biodiversidad; Bosque pluvial tropical.

\begin{abstract}
In this study we identified 221 plant species employed by the villagers from Doña Josefa community placed in Atrato town (Chocó, Colombia). We assessed nine categories of use: food, artisanal, medicinal, ornamental, toxic, magical-religious, fuel, dye and production of latex or exudates. The information was compiled taking into account the traditional knowledge of the villagers. For this purpose, we conducted semi- structured surveys and field trips with villagers with a high degree of understanding on the issue. The botanical specimens were collected, taxonomically determined and deposited in the Herbarium [CHOCO]. The most representative categories in terms of number of species were medicinal $(50,6 \%)$, food $(22,6 \%)$, fuel $(13,1 \%)$ and artisanal use $(10,4 \%)$, highlighting Malvaceae, Arecaceae, Asteraceae, Lamiaceae, Rubiaceae, Fabaceae/Mimosoideae, Gesneriaceae, Sapotaceae, Moraceae and Piperaceae families. This evidences the importance of the non-timber forest products (NTFPs) for the community of Doña Josefa, where they are mainly sources of food, medicines, handicrafts and other products with great potential for economic development, if managed sustainably.
\end{abstract}

Keywords: Use categories; NTFP; Atrato communities; Biodiversity; Tropical rainforest.

\footnotetext{
Universidad Industrial de Santander, Bucaramanga, Colombia. e-mail:angelikmac@gmail.com

Grupo de investigación en Recursos Vegetales. Línea de diversidad nativa. Universidad Tecnológica del Chocó «Diego Luis Córdoba», Quidbó, Colombia. e-mail: garciacossiof@yahoo.es

Fecha recepción: Noviembre 16, $2012 \quad$ Fecha aprobación: Diciembre 22, 2012
} 


\section{Introducción}

Es claro que hay una fuerte relación entre destrucción de bosques tropicales y disminución de diversidad biológica, así como de pérdida de la diversidad cultural y conocimientos ancestrales de los pueblos asentados en dichas zonas (Ramírez, 2007).

Por tal razón, en las últimas décadas el trabajo de los etnobotánicos se ha centrado en la necesidad de catalogar el conocimiento de plantas en una carrera contra la rápida desaparición de los recursos naturales, sobre todo en los bosques tropicales. Estos bosques, por su gran biodiversidad, son los ecosistemas con mayor potencial de productos forestales no maderables (PFNM), definidos por la Organización de las Naciones Unidas como «los bienes vegetales de origen biológico diferentes de la madera aserrada, la leña y el carbón vegetal», por ejemplo: frutos, semillas, cortezas, resinas, hojas, raíces, etc.

La región del Chocó, desde el punto de vista físico natural, es considerada el epítome de la selva húmeda tropical (Gentry, 1990) y de acuerdo con la terminología de las biozonas de Holdridge, es el único lugar del neotrópico en donde se encuentra el verdadero «bosque pluvial tropical». Cabe resaltar que según el Fondo Mundial para la Naturaleza (WWF), sólo en el Chocó biogeográfico se encuentran 7000 a 8000 especies de plantas, muchas de ellas utilizadas por las comunidades de la región como tóxicas, aromáticas, colorantes, medicinales, artesanales y alimenticias (Ramírez et al., 2003).

Parte de dichas comunidades en su afán de obtener recursos madereros, mineros y nuevas tierras para la agricultura, han hecho uso irresponsable de sus recursos naturales, olvidando por completo el papel que los PFNM juegan en la economía de los países en vía de desarrollo, en donde proporcionan insumos e ingresos a numerosos hogares urbanos y rurales (Alexiades y Shaley, 2004) y contribuyen al alivio de la pobreza de las comunidades dependientes de estos productos (FAO, 1995; Falconer, 1996).

El estudio de las plantas útiles en el corregimiento de Doña Josefa surge de la necesidad de conocer y evaluar los potenciales de la vegetación en esta región de bosque tropical, para de esta forma contribuir al conocimiento de los recursos naturales y brindar información que pueda ser utilizada en posibles planes de conservación, uso y manejo sustentable de los PFNM.

\section{Metodología}

Área de estudio. El trabajo de investigación se realizó en el corregimiento de Doña Josefa, uno de los principales centros poblados del municipio de Atrato después de la cabecera municipal. Está situado sobre la margen derecha del río Atrato, a los $5^{\circ}$ $33^{\prime}$ '08" de latitud norte y $76^{\circ} 38^{\prime}$ '96" de longitud oeste. Posee una población de 1,112 habitantes, en un área de $21.1 \mathrm{~km}^{2}$. Según la división político administrativa del municipio, limita al norte con el corregimiento de Samurindó, al oriente y al sur con Yuto cabecera municipal, y al occidente con el municipio de Río Quito. Se encuentra a una altitud de $32 \mathrm{~m}$, con una precipitación promedio anual de 8,000 mm y una temperatura promedio de $28^{\circ} \mathrm{C}$. Su territorio está constituido en su mayor parte por llanura y terreno selvático (esquema de ordenamiento territorio municipio de Atrato 2004-2015). La población del corregimiento de Doña Josefa, está integrada principalmente por afrodescendientes, quienes son nativos de este lugar o procedentes de municipios aledaños que por circunstancias como desplazamiento forzoso o el auge de explotación de recursos naturales han poblado la región. Entre las principales actividades económicas realizadas por los pobladores se encuentran la agricultura, la minería y la extracción de madera (CERPA, 1995).

Estudio etnobotánico. La información etnobotánica se recopiló durante los meses de enero a julio de 2009, mediante la aplicación de entrevistas semi-estructuradas dirigidas a agricultores, mineros, amas de casa y curanderos, debido a que las personas dedicadas a este tipo de actividades son las que tienen un contacto más directo con la vegetación implicada en amortiguar sus necesidades básicas (Figura 1).

Se entrevistaron a 48 pobladores correspondientes a 30 mujeres y 18 hombres con un rango de edad entre los 30 y 80 años. Se indagó sobre nombres vulgares, partes usadas, formas de empleo y aplicaciones de estas plantas. Posterior a esto, se realizaron recorridos en las áreas de bosque con el acompañamiento del señor Ángel Antonio Córdoba Palacios, médico tradicional de la región, para colectar ejem- 


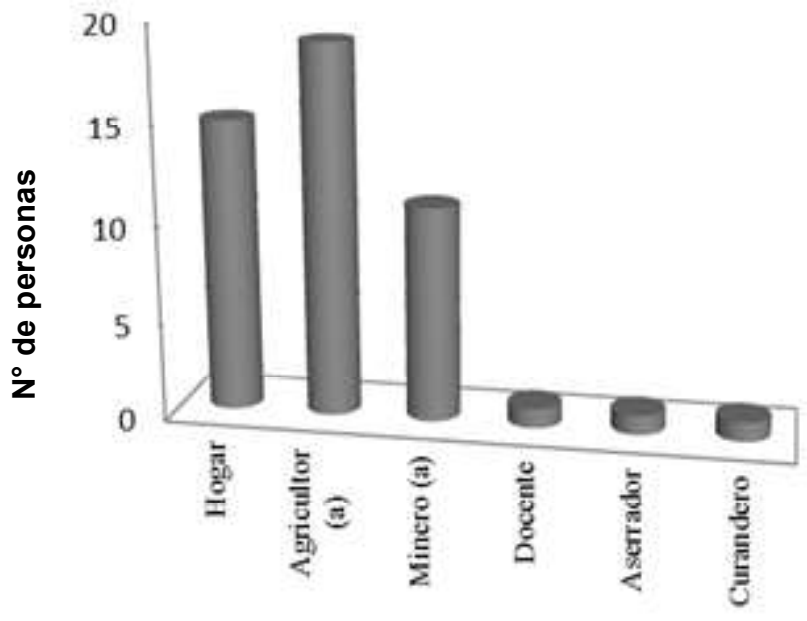

Figura 1. Ocupaciones de los pobladores que hicieron parte del estudio

plares botánicos de especies identificadas como útiles, de acuerdo con las categorías de uso que se establecieron previamente (alimento, medicinal, ornamental, tóxica, mágico-religiosa, combustible, colorantes y plantas productoras de látex o exudados).

La determinación taxonómica de los ejemplares botánicos se realizó en el Herbario [CHOCO], mediante el uso de claves taxonómicas: Gentry (1993), Mahecha (1997), Fernández-Alonso (1998). También por medio de guías ilustradas, ayuda de especialistas y colección general de especímenes. Finalmente, después del proceso de secado y montaje, el material vegetal se depositó en el Herbario [CHOCO].

Análisis estadístico. Se analizó descriptivamente el porcentaje de las familias más representativas dentro de cada una de las categorías. Para determinar el valor cultural de una especie, se utilizó el Índice de Valor de Uso (IVUs) desarrollado por Adu-Tutu
(1979) Phillips y Gentry (1993), Phillips (1996) que se establece de acuerdo con el grado de consenso en las respuestas de los informantes, con respecto a la utilidad de una especie. Este índice se estimó utilizando la expresión:

$$
\text { IVUs }=\frac{\sum \mathrm{i} U V i s}{\mathrm{Ns}}
$$

Donde: UVis = número de usos mencionados por cada informante (i), para cada especie

Ns: número de informantes entrevistados

\section{Resultados}

Se registraron un total de 221 especies distribuidas en 76 familias (Anexo 1), en donde se destacan por el número de especie las familias Malvaceae, Arecaceae, Asteraceae, Lamiaceae, Rubiaceae, Fabaceae/Mimosoideae, Gesneriaceae, Sapotaceae, Moraceae y Piperaceae (Figura 2).

El mayor número de especies lo presenta la categoría medicinal y alimenticia, le sigue en orden descendiente, combustible, artesanal, ornamental, plantas productoras de látex o exudado, mágico-religiosas, tóxicas y colorantes, siendo estas dos últimas las de menor representación en el estudio (Figura 3).

También cabe resaltar que muchas especies se registran en más de una categoría, debido a sus múltiples usos, tal es el caso de Attalea allenii, Manicaria saccifera, Hymenaea oblongifolia y Sacoglottis procera.

De las especies con potencialidades de uso, el mayor corresponde a las utilizadas con fines curativos. Dentro de las 53 familias que se registran como medicinales, se destacan Asteraceae con $8 \%$ de las

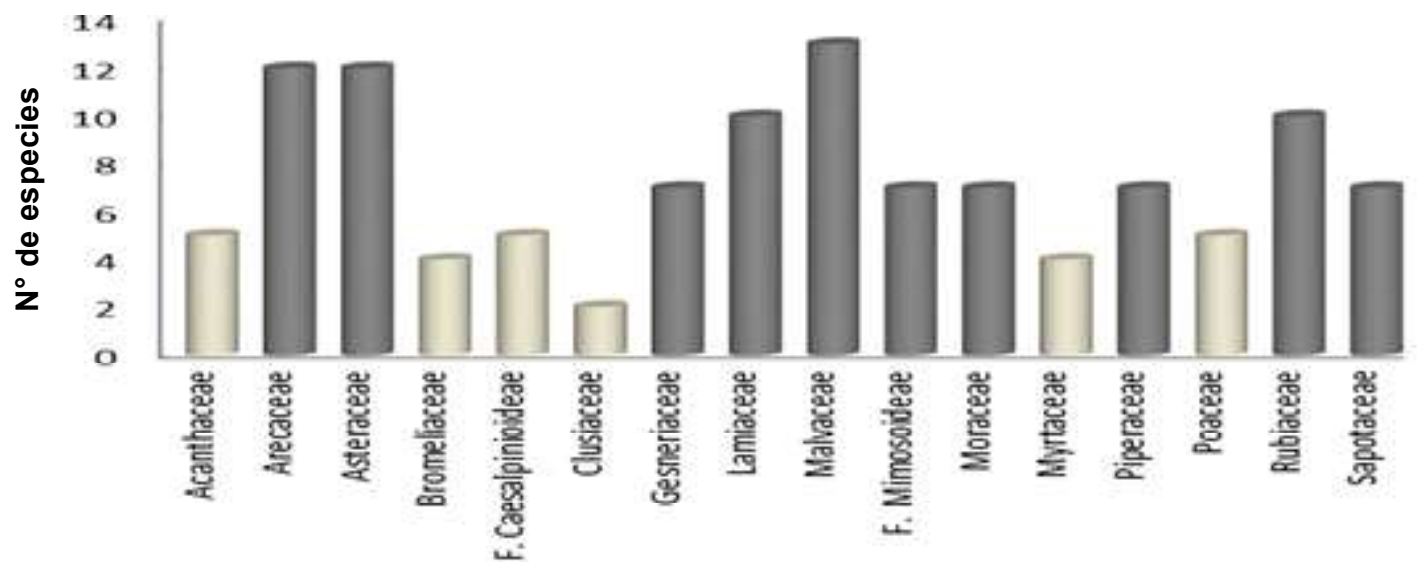

Figura 2. Familias con mayor número de especies en todo el estudio 
especies, seguida de Lamiaceae y Gesneriaceae con 6.3\%, Malvaceae y Piperaceae con el $5.4 \%$ y finalmente Acanthaceae y Rubiaceae con el $4.5 \%$ cada una (Figura 4). Los valores de uso más altos corresponden a Sida rhombifolia (0.64), Glossoloma panamense (0.58), Columnea consanguinea (0.52), Apeiba membranacea (0.46) y Scoparia dulcis (0.29). La parte más usada es la hoja, las dolencias más tratadas son parásitos intestinales, sarpullido, dolor de cabeza, cólicos abdominales y mordeduras de serpientes y las formas de empleo más comunes son en cocciones, baños, emplastos e infusiones.

Dentro de las 25 familias de plantas usadas en la categoría alimenticia, las que registran el mayor porcentaje de especies son Arecaceae (22\%), Malvaceae (10\%) y Fabaceae/Mimosoideae, Rubiaceae y Sapotaceae (6\% cada una) (Figura 5). Es importante destacar que el IVU más alto lo reportaron Attalea allenii (0.85), Oenocarpus bataua (0.83), Bactris gasipaes (0.60) y Oenocarpus mapora (0.48) evidenciando la importancia que toman algunas especies de palmas en la alimentación de los habitantes de esta comunidad. Las formas más comunes de preparación son en jugos, ensaladas y guisos.

En la categoría artesanal se encuentran 23 especies distribuidas en 11 familias en

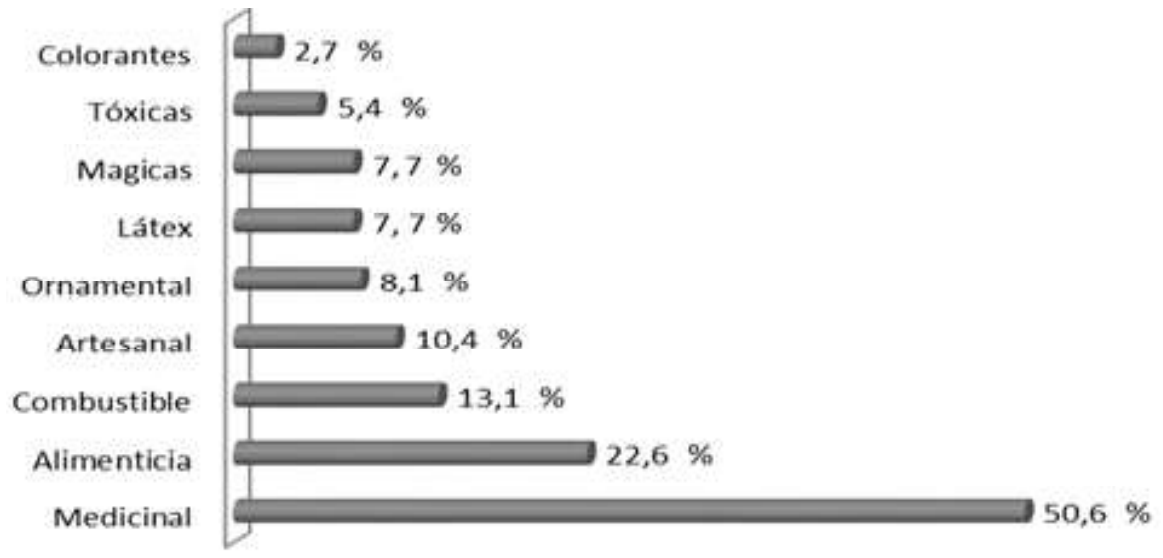

Figura 3. Porcentaje de especies dentro de las 9 categorías de uso.

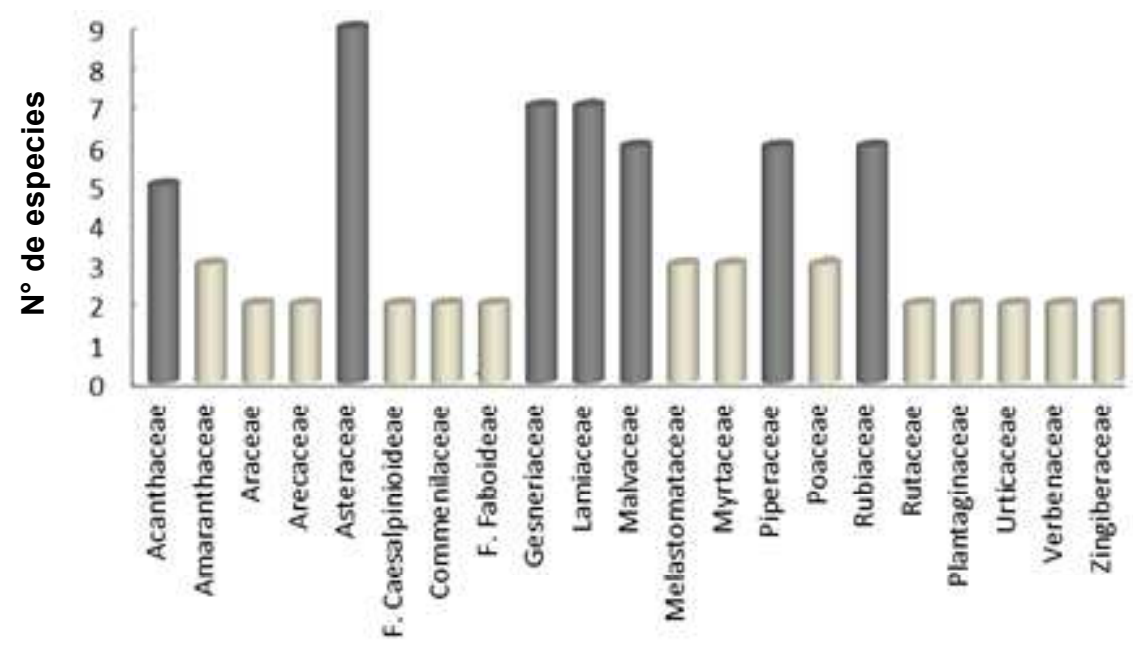

Figura 4. Familias con mayor número de especies en la categoría medicinal

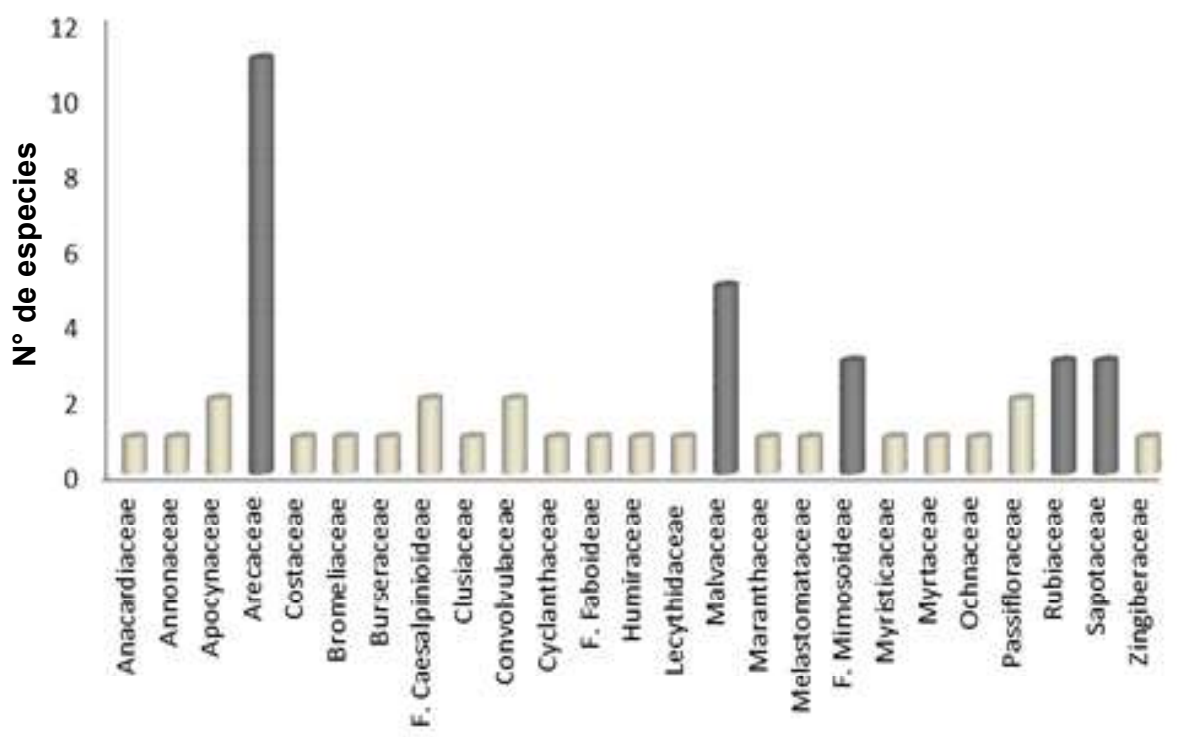

Figura 5. Familias con mayor número de especies en la categoría alimenticia 


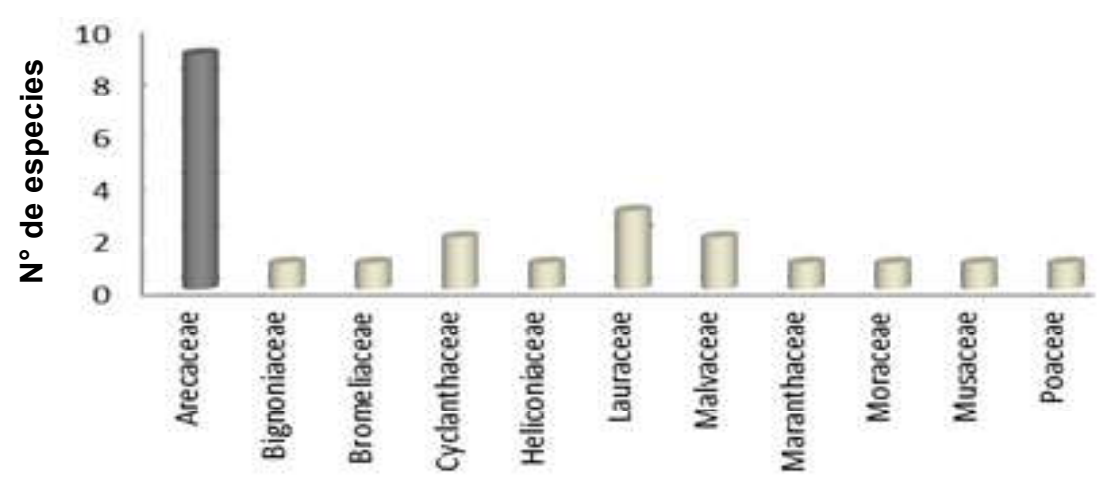

Figura 6. Familias con mayor número de especies en la categoría artesanal.

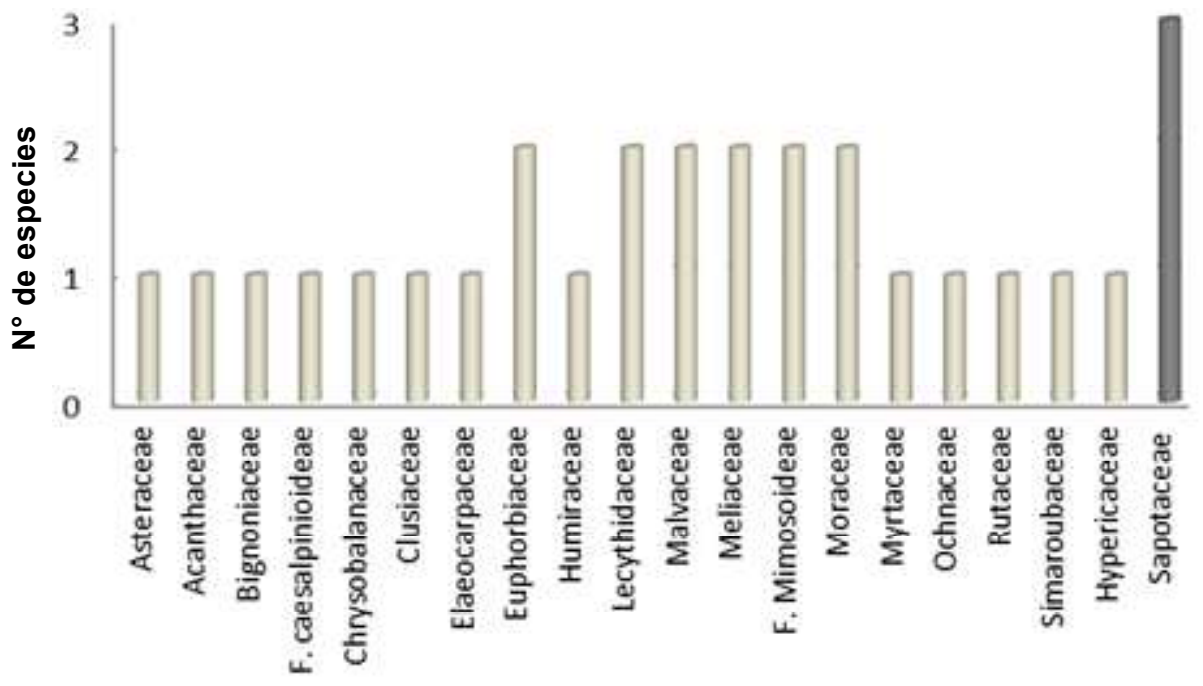

Figura 7. Familias con mayor número de especies en la categoría combustible.

donde predomina Arecaceae con el 39.1\% de las especies registradas (Figura 6); sin embargo, el valor de uso más alto lo presenta Carludovica palmata perteneciente a la familia Cyclanthaceae, en donde el total de los pobladores entrevistados la identifican como de gran potencial en la fabricación de diferentes artesanías. Otras especies que sobresalen por su uso artesanal son Manicaria saccifera, Ischnosiphon arouma, Thoracocarpus bissectus, Astrocaryum standleyanum y Heliconia sp. La parte más usada es el cogollo (brote joven de cualquier vegetal) de la planta y los productos que más elaboran son los sombreros, esteras, cestas y escobas.

La categoría de plantas usadas como combustible reúne un importante grupo de especies vegetales con potencial para ser usadas en la generación de energía térmica en la región (Figura 7). Sobresalen las especies Luehea seemannii, Jacaranda hesperia, Pouteria caimito, Inga sp., Licania macrocarpa, Eschweilera pittieri, Simarouba amara y Maquira sp. Cabe resaltar que estas especies por lo general no son comercializadas siendo empleadas para uso doméstico dentro de cada núcleo familiar.

Dentro de las plantas de ornato se citan 15 especies entre las que se encuentran Palicourea guianensis, Hedychium coronatum, Hibiscus rosea, Anthurium sp. y Heliconia sp., usadas sobre todo en la decoración de fiestas patronales debido a la presencia de flores llamativas.

En la categoría de plantas productoras de látex se registran 10 familias, sobresaliendo Moraceae y Sapotaceae con el mayor número de especies. Las más importantes en cuanto al valor de uso son Couma macrocarpa, Brosimum utile, Vismia macrophylla y Artocarpus communis, que además presentan usos medicinales.

En las restantes categorías como colorantes, tóxicas y mágico-religiosas, se registran los porcentajes más bajos en cuanto al número de especies, pero se destacan los altos valores de uso que presentan Bixa orellana y Genipa americana (colorantes), Phyllanthus acuminatus (tóxica), Petiveria alliacea y Ruta graveolens (mágico-religiosas).

Debido a que no se pudo colectar el material vegetal, en algunas especies no fue posible su determinación taxonómica, por lo tanto sólo se reporta su nombre común. Tal es el caso de clavel, grama matojo, grama gateadora en la categoría medicinal, mano de león en la categoría ornamental, manteco en la categoría combustible, palo de ají en la categoría de tóxico y pega paruma en la categoría de planta mágico-religiosa.

También se resalta que 
en este estudio se quería hacer énfasis en la flora silvestre, sin embargo se reportan especies cultivadas y semicultivadas que son de gran importancia para la comunidad.

\section{Discusión}

Se resalta que las plantas medicinales son de gran importancia para los habitantes de la comunidad de Doña Josefa, quienes antes de recurrir a medicamentos convencionales hacen uso de los recursos naturales de origen vegetal para tratar sus problemas de salud, esto también se evidencia en el estudio de PinoBenítez (2000) realizado en la comunidad de Quibdó, Chocó.

El número de especies reportadas en la categoría medicinal, es similar a la encontrada por Caballero (1995) en la región del río Patía donde las etnias negras e indígenas utilizan 110 especies para fines curativos, y al de Pino-Benítez y Valois (2004) realizado en el municipio de Quibdó, donde se identificaron 135 especies de plantas medicinales. En cuanto a Columnea consanguinea, Columnea picta, Drymonia killipii, Psychotria poeppigiana, Psychotria cooperi y Coccocypselum lanceolatum cabe mencionar que se reportan como especies de grandes potencialidades medicinales en el departamento del Chocó, al igual que lo reportado por Pino-Benítez (2006).

Dentro de la categoría alimenticia se presenta una gran diversidad de palmas y se destaca el potencial de Oenocarpus bataua ya que es considerada una especie promisoria, por sus frutos ricos en aceite y proteínas de alta calidad (Galeano y Bernal, 1987; Balick, 1992; Morcote-Ríos et al., 1998).

En cuanto a las plantas útiles para la elaboración de artesanías, la familia Arecaceae reúne al mayor número de especies, dato que coincide con los resultados de Pino-Benítez y Valois (2004) en el municipio de Quibdó y por Feuillet et al. (2011) en el departamento del Cauca, Colombia.

Las plantas colorantes tuvieron poca representación respecto al número de especies usadas para tal fin, pero se destaca la extracción artesanal y las potencialidades de aprovechamiento de Genipa americana tal como lo propone Pino-Chalá et al. (2003) para el municipio de Quibdó.
En la categoría de plantas de ornato sobresalen las heliconias debido a sus brácteas de colores. Cabe resaltar que la región pacífica colombiana posee el $37 \%$ de las especies reportadas en todo el país, con un alto grado de endemismo y que estas plantas tienen grandes potencialidades de uso como flores de corte (Kress et al., 1999).

Finalmente, es importante mencionar que dentro del estudio la familia Arecaceae es la de mayor representación en varias categorías, debido a que las palmas son reconocidas ampliamente por su gran diversidad de usos en bosques tropicales (PinedoVásquez et al., 1990; Phillips y Gentry 1993, Prance et al., 1987).

\section{Conclusiones}

El diagnóstico de los PFNM utilizados en esta comunidad indica una alta diversidad de especies, lo que representa un conocimiento invaluable que se debe promover y proteger.

Se debe enfatizar en las especies que presentan múltiples usos porque se pueden ver sometidas a mayores presiones por su forma de aprovechamiento, como es el caso del Cabecinegro y el Táparo.

Los bosques tropicales son una importante fuente de PFNM, y contribuyen a la diversificación de las economías locales, por lo tanto deben ser manejados de manera racional y amigable con el medio ambiente. A pesar de que el índice de valor de uso es una herramienta informativa, sus resultados se pueden afectar por la cantidad de tiempo invertido y el número de personas encuestadas, pues para obtener datos más confiables es necesario hacer re-entrevistas y de esta forma no subestimar cifras.

\section{Agradecimientos}

A los habitantes del corregimiento de Doña Josefa por su hospitalidad, en especial a Didier Orlando Palacios por la ayuda prestada en el trabajo de campo y a Jesús Antonio Córdoba por sus sabias enseñanzas en cuanto al uso de las plantas medicinales. A la Universidad Tecnológica del Chocó, al grupo de Investigación en Recursos Vegetales y al personal adscrito al Herbario CHOCO por sus aportes en la realización de este trabajo. 


\section{Literatura citada}

Adu-Tutu M, Afful Y, Asante-Appiah K, Leberman D, Hall JB, Elvin-Lewis M. 1979. Chewing stick usage in southern Ghana. Economic Botany. 33 (3): 320-8.

Alcaldía Municipal del Atrato. Instituto de Investigaciones Ambientales del Pacífico. Convenio BID-Plan PacíficoMAVDT-Gobernación del Chocó-UTCH-IIAP. Esquema de ordenamiento territorial del municipio de Atrato, Chocó. 2004-2015.

Alexiades M, Shanley P. 2004. Productos forestales, medios de subsistencia y conservación. Estudio de caso sobre sistemas de manejo de productos forestales no maderables. Centro para la Investigación Forestal Internacional. 3: 1-22.

Balick MJ. 1992. Jessenia y Oenocarpus: palmas aceiteras neotropicales dignas de ser domesticadas. Estudio FAO: producción y protección vegetal. Roma: FAO.

Caballero MR. 1995. La etnobotánica en las comunidades negras e indígenas del Delta del río Patía. Colección biblioteca Abya-Yala $N^{\circ}$ 26. Cayambe: Ediciones Abya-Yala,.

CERPA (Centro de Estudios Regionales del Pacífico). 1995. Prioridades socioambientales del departamento del Chocó. Documento técnico. Quibdó: CERPA.

Falconer J. 1996. Developing research frames for non-timber forest products: experience from Ghana. . In: Ruiz Pérez M, Arnold JEM (eds.). Current issues in non-timber forest products research. Bogor; Centre for International Forestry Research. Pp. 143-60.

FAO. The Food and Agriculture Organisation of the United Nations. 1995. Non wood forest products for rural income and sustainable forestry. Rome: NWFPs 7.

Fernández-Alonso JL. 1998. Novedades taxonómicas, nomenclaturales y corológicas en el género Pachira Aubl. (Bombacaceae). An Jard Bot. Madrid 56 (2): 305-14.

Feuillet C, Macias D, Cerón E. 2011. Plantas útiles para la elaboración de artesanías en el departamento del Cauca (Colombia). Bol Cient Centro de Museos. 15 (2): 40-59.

Galeano G, Bernal R. 1987. Palmas del departamento de Antioquia. Región occidental. Bogotá: Universidad Nacional de Colombia.

Gentry A. 1990. Selva húmeda tropical. En: Villegas B. (ed.) Selva húmeda de Colombia. Bogotá: Villegas Editores. p. 13-52.

Gentry A. 1993. A field guide to the families and genera of woody pants of northwest South America. Conservation International. $895 \mathrm{pp}$.

Kress W, Betancur J, Echeverri B. 1999. Heliconias llamaradas de la selva colombiana. Bogotá: Ed. Cristina Uribe.
Phillips O, Gentry A. 1993. The useful plants of Tambopata, Peru: I. Statistical hypothesis tested with a new quantitative technique. Econ Bot. 47 (1): 15-32.

Phillips O. 1996. Some quantitative methods for analyzing ethnobotanical knowledge. En: Alexiades M (ed.). Selected guidelines for ethnobotanical research: a field manual. New York: The New York Botanical Garden,. pp. 171-97.

Pinedo-Vásquez M, Zarin D, Jipp P, Chota-Inuma J. 1990. Usevalues of tree species in a communal forest reserve in northeast Peru. Conservat Biol. 4 (4): 405-17.

Pino-Benítez N. 2000. Panorámica de algunas plantas medicinales del municipio de Quibdó. Rev Institucional, Universidad Tecnológica del Chocó, 13: 25-36.

Pino-Benítez N, Valois H. 2004. Ethnobotany of four black communities of the Municipality of Quibdó-Chocó, Colombia. Lyonia. 7: 59-68.

Pino-Benítez N. 2006. Botánica y screening fitoquímico de doce plantas usadas en medicina tradicional en el departamento del Chocó, Colombia. Revi Latinoam Recs Nat. 2 (1): 33-44.

Pino-Chalá W, Guerrero J, Castro A, Palacios J, Castro A. 2003. Extracción artesanal de colorantes naturales, una alternativa de aprovechamiento de la diversidad biológica del chocó, Colombia. Acta Biol Colomb., 8 (2): 95-8.

Prance GT, Balee W, Boom B, Carneiro RL. 1987. Quantitative ethnobotany and the case for conservation in Amazonia. Conserv Biol. 1 (4): 296-310.

Mahecha G. 1997. Fundamentos y metodologías para la identificación de plantas. Proyecto Biopacífico, Ministerio del Medio Ambiente, GEF-PNUD. Bogotá: Lerner Ltda. 282 pp.

Morcote-Ríos G, Cabrera-Becerra G, Mahecha-Rubio D, FrankyCalvo CE, Cavelier IF. 1998. Las palmas entre los grupos cazadores-recolectores de la Amazonía colombiana. Caldasia. 20: 57-74.

Ramírez C. 2007. Etnobotánica y la pérdida de conocimiento tradicional en el siglo 21. Ethnobot Res Applic. 5: 241-4.

Ramírez Y, Copete S, Lozano N, Lagarejo E, Arroyo J, García F,et al. 2003. Etnobotánica de las especies arbóreas presentes en la parcela permanente de investigación en biodiversidad (PPIB) en Salero, Unión Panamericana, Chocó. pp. 69-76. En: García F, Ramos Y, Palacios J, Arroyo J, Mena A, Gonzáles M. (eds.). Salero: Diversidad biológica de un bosque pluvial tropical. Quibdó: Universidad Tecnológica del Chocó «Diego Luis Córdoba», Instituto de Investigaciones Ambientales del Pacífico, Comunidad de Salero.125 pp.

WWF. [en línea] http://www.wwf.org.co/donde_trabajamos/ choco_darien/riquezas/ 
Anexo 1. Lista de las especies de PFNM utilizadas en el corregimiento Doña Josefa, Chocó, Colombia, con sus respectivos nombres vulgares y valores de uso por categoría

\begin{tabular}{|c|c|c|c|c|c|}
\hline \multirow[t]{2}{*}{ No } & \multicolumn{2}{|c|}{ Nombre } & \multirow[t]{2}{*}{ Familia } & \multirow[t]{2}{*}{ Usos } & \multirow[t]{2}{*}{ IVU } \\
\hline & vulgar & científico & & & \\
\hline 1 & Aceite & Calophyllum mariae Planch. y Triana & Calophyllaceae & Combustible & 0.02 \\
\hline 2 & Ajengibre & Zingiber officinale Roscoe. & Zingiberaceae & Alimento-Medicinal & $0.02-0.10$ \\
\hline 3 & Albacón & Scutellaria pseudocoleus Fern. Alonso & Lamiaceae & Ornamental & 0.02 \\
\hline 4 & Albahaca (las 7) & Ocimum sp. & Lamiaceae & Mágico religiosa & 0.33 \\
\hline 5 & Algarrobo & Hymenaea oblongifolia Huber & Fabacaeae/Caesalpiniodeae & $\begin{array}{l}\text { Alimento-Combustible } \\
\text { Mágico religiosa-Látex }\end{array}$ & $\begin{array}{r}0.46-0.08 \\
0.02-0.02\end{array}$ \\
\hline 6 & Algodoncillo & Croton killipianus Croizat & Euphorbiaceae & Combustible & 0.06 \\
\hline 7 & Aliso & Pollalesta discolor (Kunth) Aristeg. & Asteraceae & Combustible & 0.31 \\
\hline 8 & Almirajó de monte & Matisia dowdingii Sprague. & Malvaceae/Bombacoideae & Alimento & 0.17 \\
\hline 9 & Amansa guapo & Justicia pectoralis Jacq. & Acanthaceae & Medicinal & 0.02 \\
\hline 10 & Amansa justicia & Justicia $\mathrm{sp}$ & Acanthaceae & Medicinal-Mágico religiosa & $0.06-0.02$ \\
\hline 11 & Amaranto & Iresine herbstii Hook. ex Lindl. & Amaranthaceae & Medicinal & 0.12 \\
\hline 12 & Amargo (palma) & Welfia georgii $\mathrm{H}$. Wendl. & Arecaceae & Artesanal & 0.06 \\
\hline 13 & Amargo & & Rubiaceae & Medicinal & 0.02 \\
\hline 14 & Amargosa & Peperomia pernambucensis Miq. & Piperaceae & Medicinal & 0.04 \\
\hline 15 & Anamú & Petiveria alliacea L. & Phytolaccaceae & Medicinal-Mágico religiosa & $0.06-0.25$ \\
\hline 16 & Árbol del pan & Artocarpus communis J.R.Forst. y G.Forst. & Moraceae & $\begin{array}{l}\text { Medicinal-Látex } \\
\text { Alimento }\end{array}$ & $\begin{array}{r}0.02-0.42 \\
0.16\end{array}$ \\
\hline 17 & Artemisa & Ambrosia artemisiifolia L. & Asteraceae & Medicinal & 0.10 \\
\hline 18 & Aserrín/Capitancillo & Pentaclethra macroloba (Willd.) Kuntze & Fabaceae/Mimosoideae & Tóxica & 0.25 \\
\hline 19 & Azafrán & Crocus sativus $\mathrm{L}$. & Iridaceae & Alimento-Medicinal & $0.02-0.02$ \\
\hline & & & & Colorante & 0.06 \\
\hline 20 & Azucena & Lilium sp. & Liliaceae & Ornamental & 0.04 \\
\hline 21 & Bacao & Theobroma bicolor Humb & Malvaceae/ Byttnerioideae & Alimento & 0.04 \\
\hline 22 & Badea & Passiflora quadrangularis $\mathrm{L}$. & Passifloraceae & Medicinal & 0.02 \\
\hline 23 & Badea de monte & Passiflora vitifolia Kunth & Passifloraceae & Alimento & 0.14 \\
\hline 24 & Balsamina & Momordica charantia L. & Cucurbitaceae & Medicinal & 0.02 \\
\hline 25 & Balso & Ochroma piramidale (Cav.ex Lam.)Urb. & Malvaceae/Bombacoideae & Artesanal & 0.02 \\
\hline 26 & Barbasco & Lonchocarpus sp. & Fabaceae/Faboideae & Tóxica & 0.37 \\
\hline 27 & Batata & Ipomoea batata $\mathrm{L}$. & Convolvulaceae & Alimento-Medicinal & $0.02-0.02$ \\
\hline 28 & $\begin{array}{l}\text { Bejuco del palo } \\
\text { grande }\end{array}$ & Strychnos sp & Loganiaceae & Medicinal & 0.02 \\
\hline 29 & Bejuco del sol & Aristolochia oblongata Jacq. & Aristolochiaceae & Medicinal & 0.14 \\
\hline 30 & Bejuco negro & Vernonia sp. & Asteraceae & Medicinal & 0.04 \\
\hline 31 & Bellísima & $\begin{array}{l}\text { Chrysothemis friedrichsthaliana } \\
\text { (Hanst.) H.E. Moore }\end{array}$ & Gesneriaceae & Medicinal & 0.04 \\
\hline 32 & Bija & Bixa orellana $\mathrm{L}$. & Bixaceae & Colorante & 1 \\
\hline 33 & Bledo & Amaranthus spinosus L. & Amaranthaceae & Medicinal & 0.02 \\
\hline 34 & Borojó & Borojoa patinoi Cuatrec. & Rubiaceae & Alimento-Medicinal & 0.04 \\
\hline 35 & Botoncillo & Sphyllantes sp. & Asteraceae & Medicinal & 0.12 \\
\hline 36 & Broncho de plátano & Musa sp. & Musaceae & Artesanal & 0.02 \\
\hline 37 & Cabecinegro & Manicaria saccifera Gaertn. & Arecaceae & $\begin{array}{l}\text { Alimento-Medicinal } \\
\text { Artesanal }\end{array}$ & $\begin{array}{r}0.06-0.06 \\
0.30\end{array}$ \\
\hline 38 & Cacahuillo & Herrania purpurea Pittier & Malvaceae/ Byttnerioideae & Alimento & 0.23 \\
\hline 39 & Caidita & Nectandra sp. & Lauraceae & Medicinal & 0.27 \\
\hline 40 & Caimito & Pouteria caimito Radlk. & Sapotaceae & $\begin{array}{l}\text { Alimento-Combustible } \\
\text { Látex }\end{array}$ & $\begin{array}{r}0.7-0.83 \\
0.22\end{array}$ \\
\hline 41 & Caimito de monte & Chrysophyllum sp & Sapotaceae & Alimento & 0.81 \\
\hline 42 & Caimito vela & Pouteria sp. & Sapotaceae & Combustible & 0.02 \\
\hline 43 & Caimitón & Pouteria sp. & Sapotaceae & Tóxica-Látex & $0.04-0.02$ \\
\hline 44 & Caña agria & Costus villosissimus Jacq. & Costaceae & Medicinal & 0.04 \\
\hline 45 & Caña brava & Gynerium sagittatum Aubl. & Poaceae & Artesanal & 0.02 \\
\hline 46 & Caña fistula & Cassia grandis $\mathrm{L}$. & Fabaceae/Caesalpiniodeae & Alimento & 0.02 \\
\hline 47 & Cañaguate & Dimerocostus strobilaceus Kuntze & Costaceae & Alimento & 0.04 \\
\hline 48 & Caraño & Trattinnickia aspera Standl. & Burseraceae & Alimento-Látex & 0.020 .02 \\
\hline 49 & Carbonero & Licania macrocarpa Cuatrec. & Chrysobalanaceae & Combustible & 0.83 \\
\hline 50 & Cargadera & Guatteria amplifolia Triana y Planch. & Annonaceae & Medicinal & 0.02 \\
\hline 51 & Carrá & Huberodendron patinoi Cuatrec. & Malvaceae/Bombacoideae & Combustible & 0.02 \\
\hline 52 & Cartucho & Anthurium sp. & Araceae & Ornamental & 0.02 \\
\hline 53 & Cascajero & Psychotria cooperi Standl. & Rubiaceae & Medicinal & 0.33 \\
\hline 54 & Castaño & Compsoneura atopa A.C. Smith & Myristicaceae & Alimento-Látex & $0.73-0.02$ \\
\hline 55 & Catalina & Clibadium asperum (Aubl.) DC. & Asteraceae & Tóxica & 0.54 \\
\hline 56 & Catuga & Ischnosiphon inflatus L. & Maranthaceae & Alimento & 0.02 \\
\hline 57 & Caucho & Castilla elástica Sesse ex Cerv. & Moraceae & Tóxica-Látex & $0.02-0.63$ \\
\hline 58 & Cedro & Cedrela odorata L. & Meliaceae & Combustible & 0.10 \\
\hline 59 & Cedro macho & Carapa guianensis Aubl. & Meliaceae & Combustible & 0.02 \\
\hline 60 & Cedrón & Simaba cedron Planch. & Simaroubaceae & Medicinal & 0.02 \\
\hline 61 & Celedonia & Peperomia pellucida (L.) H.B.K. & Piperaceae & Medicinal & 0.23 \\
\hline
\end{tabular}


Anexo 1. Lista de las especies de PFNM utilizadas en el corregimiento Doña Josefa, Chocó, Colombia, con sus respectivos nombres vulgares y valores de uso por categoría (continuación)

\begin{tabular}{|c|c|c|c|c|c|}
\hline \multirow[t]{2}{*}{$\mathbf{N}^{\circ}$} & \multicolumn{2}{|c|}{ Nombre } & \multirow[t]{2}{*}{ Familia } & \multirow[t]{2}{*}{ Usos } & \multirow[t]{2}{*}{ IVU } \\
\hline & vulgar & científico & & & \\
\hline 62 & Cerveza/Pavonilla & Glossoloma panamense C.V. Morton & Gesneriaceae & Medicinal & 0.58 \\
\hline 63 & Chachajo & Aniba perutilis Hemsl. & Lauraceae & Artesanal & 0.02 \\
\hline \multirow[t]{2}{*}{64} & Chanó & Sacoglottis procera (Miq.) Cuatrec. & Humiraceae & Alimento-Combustible & $0.35-0.83$ \\
\hline & & & & Látex-Tóxica & $0.02-0.20$ \\
\hline 65 & Chascarrá & Bactris barronis L.H. Bailey & Arecaceae & AlimentoArtesanal & $0.10-0.02$ \\
\hline 66 & Chirrinchao & Phyllanthus acuminatus Vahl. & Euphorbiaceae & Tóxica & 0.52 \\
\hline 67 & Choco blanco & Napeanthus apodemus & Gesneriaceae & Medicinal-Mágico religiosa & $0.02-0.02$ \\
\hline 68 & Choco morado & Napeanthus sp. & Gesneriaceae & Medicinal-Mágico religiosa & $0.02-0.02$ \\
\hline 69 & Chocolate biche & Theobroma cacao L. & Malvaceae/ Byttnerioideae & Medicinal & 0.02 \\
\hline 70 & $\begin{array}{l}\text { Chocolatina de } \\
\text { monte }\end{array}$ & Theobroma glaucum Karst. & Malvaceae/ Byttnerioideae & Alimento & 0.06 \\
\hline 71 & Chontaduro & Bactris gasipaes Humb. y Bonpl. ex Kunth & Arecaceae & Alimento-Medicinal & $0.60-0.02$ \\
\hline 72 & Choibá & Dipteryx oleifera Benth. & Fabaceae/Faboideae & Alimento & 0.02 \\
\hline 73 & Churco & Monolena cordifolia Triana & Melastomataceae & Medicinal & 0.10 \\
\hline 74 & Churco & Monolena primulaeflora Hook. & Melastomataceae & Medicinal & 0.02 \\
\hline 75 & Churima & Inga coruscans Humb. y Bonpl. ex Willd & Fabaceae/Mimosoideae & Alimento & 0.30 \\
\hline 76 & Cienpiesitos & Tonina fluviatilis Aubl. & Eriocaulaceae & Medicinal & 0.02 \\
\hline 77 & Citronela & Cymbopogon nardus(L.) Rendle & Poaceae & Medicinal-Mágico religiosa & $0.06-0.04$ \\
\hline 78 & Clavel & & & Medicinal & 0.02 \\
\hline 79 & Clavellino & Brownea macrophylla Linden ex Marst. & Fabaceae/Caesalpiniodeae & Ornamental & 0.02 \\
\hline 80 & Col & Brassica oleraceae L. & Brassicaceae & Medicinal & 0.02 \\
\hline 81 & Cólera & Cassia sp. & Fabaceae/Caesalpiniodeae & Medicinal & 0.02 \\
\hline 82 & $\begin{array}{l}\text { Corbatica o } \\
\text { peperreme }\end{array}$ & Anthurium sp. & Araceae & Medicinal & 0.02 \\
\hline 83 & $\begin{array}{l}\text { Cordoncillo/ } \\
\text { pipilongo }\end{array}$ & Piper tuberculatum Jacq & Piperaceae & Medicinal & 0.10 \\
\hline 84 & Corocito & Bractis sp. & Arecaceae & Alimento & 0.02 \\
\hline 85 & Coronillo & Bellucia axinanthera Triana & Melastomataceae & Alimento-Medicinal & $0.83-0.06$ \\
\hline 86 & Corozo & Bractis coloradonis L.H. Bailey & Arecaceae & Alimento & 0.04 \\
\hline 87 & Cuerito & Alchornea sp. & Euphorbiaceae & Combustible & 0.06 \\
\hline 88 & Desbaratadora & Drymonia killipii Wiehl. & Gesneriaceae & Medicinal & 0.23 \\
\hline 89 & Don Pedrito & Oenocarpus mapora H. Karst. & Arecaceae & Alimento-Artesanal & $0.48-0.02$ \\
\hline 90 & Doña Juana & Adenostemma lavenia (L.) Kuntze & Asteraceae & Medicinal & 0.04 \\
\hline 91 & Doradilla & Oxalis barrelieri $\mathrm{L}$. & Oxalidaceae & Medicinal & 0.04 \\
\hline 92 & Dormilón & Pentaclethra macroloba (Willd.) Kuntze. & Fabaceae/Mimosoideae & Tóxica & 0.08 \\
\hline 93 & $\begin{array}{l}\text { Escancel/ } \\
\text { Sangrinaria }\end{array}$ & Alternanthera $\mathrm{sp}$ & Amaranthaceae & Medicinal-Colorante & $0.35-0.02$ \\
\hline 94 & Escobabosa & Sida rhombifolia L. & Malvaceae/Malvoideae & Medicinal & 0.64 \\
\hline 95 & Escubilla & Scoparia dulcis L. & Plantaginaceae & Medicinal & 0.29 \\
\hline 96 & Espadilla/chundul & Kyllinga pumila Michx. & Cyperaceae & Medicinal & 0.08 \\
\hline 97 & Flor de mayo & Palicourea guianensis Aubl. & Rubiaceae & Ornamental & 0.10 \\
\hline 98 & Flor del muerto & Baccharis sp. & Asteraceae & Medicinal-Ornamental & $0.02-0.02$ \\
\hline 99 & Gallinaza & Tagetes apetala Posada & Asteraceae & Medicinal & 0.06 \\
\hline 100 & Galve & Senna reticulata Willd. & Fabaceae / Caesalpiniodeae & Medicinal & 0.08 \\
\hline 101 & Grama de matojo & & Poaceae & Medicinal & 0.08 \\
\hline 102 & Grama gateadora & & Poaceae & Medicinal & 0.02 \\
\hline 103 & Granadilla de monte & Passiflora vitifolia Kunth. & Passifloraceae & Alimento & 0.19 \\
\hline 104 & Guácimo blanco & Apeiba membranacea Spruce ex. Benth. & Malvaceae/ Byttnerioideae & Medicinal & 0.46 \\
\hline 105 & Guácimo colorado & Luehea seemannii Triana y Planch & Malvaceae/ Grewioideae & Medicinal-Combustible & $0.06-0.85$ \\
\hline 106 & Guaco & Guzmania musaica (Linden y André) Mez. & Bromeliaceae & Medicinal & 0.04 \\
\hline 107 & Guama de monte & Inga sp. & Fabaceae/Mimosoideae & Alimento & 0.04 \\
\hline 108 & Guamo & Inga sp. & Fabaceae/Mimosoideae & Alimento-Combustible & $0.20-0.85$ \\
\hline 109 & $\begin{array}{l}\text { Guanábana de } \\
\text { monte }\end{array}$ & Anonna sp. & Annonaceae & Alimento & 0.02 \\
\hline 110 & Guasca peo & Couratari guianensis Aubl. & Lecythidaceae & Combustible & 0.02 \\
\hline 111 & Guasca/o & Eschweilera pittieri R. Knuth & Lecythidaceae & Combustible & 0.50 \\
\hline 112 & Guayaba & Psidium guajava $\mathrm{L}$. & Myrtaceae & Medicinal & 0.02 \\
\hline 113 & Guayaba agria & Psidium friedrichsthalianum (O. Berg) Nied. & Myrtaceae & Medicinal & 0.02 \\
\hline 114 & Guayabo & Eugenia sp. & Myrtaceae & Combustible & 0.08 \\
\hline 115 & Guerregue & Astrocaryum standleyanum L. H. Bailey & Arecaceae & Alimento-Artesanal & $0.04-0.25$ \\
\hline 116 & Guinea & Sanchezia sp. & Acanthaceae & Medicinal & 0.02 \\
\hline 117 & Hierba buena & Mentha spicata L. & Lamiaceae & Medicinal-Mágico religiosa & $0.27-0.02$ \\
\hline 118 & Hierba de Adán & Irlbachia alata (Aubl.) Maas & Gentianaceae & Medicinal & 0.02 \\
\hline 119 & Hierba de sapo & $\begin{array}{l}\text { Conobea scoparioides (Cham. y } \\
\text { Schltdl.) Benth. }\end{array}$ & Plantaginaceae & Medicinal-Mágico religiosa & $0.10-0.02$ \\
\hline 120 & $\begin{array}{l}\text { Hierba del } \\
\text { carpintero }\end{array}$ & Selaginella erythropus Spring. & Selaginellaceae & Medicinal-Mágico religiosa & $0.20-0.04$ \\
\hline
\end{tabular}


Anexo 1. Lista de las especies de PFNM utilizadas en el corregimiento Doña Josefa, Chocó, Colombia, con sus respectivos nombres vulgares y valores de uso por categoría (continuación)

\begin{tabular}{|c|c|c|c|c|c|}
\hline \multirow[t]{2}{*}{ No } & \multicolumn{2}{|c|}{ Nombre } & \multirow[t]{2}{*}{ Familia } & \multirow[t]{2}{*}{ Usos } & \multirow[t]{2}{*}{ IVU } \\
\hline & vulgar & científico & & & \\
\hline 121 & Hierba dulce & Borreria latifolia (Aubl.) K. Schum. & Rubiaceae & Alimento & 0.02 \\
\hline 122 & Hierba la lora & Trichomanes elegans Rich. & Hymenophyllaceae & Medicinal & 0.04 \\
\hline 123 & Hierba santa & Kalanchoe pinnata (Lam.) Pers. & Crassulaceae & Medicinal & 0.08 \\
\hline 124 & Higuerillo & Ricinus communis $\mathrm{L}$. & Euphorbiaceae & Medicinal & 0.02 \\
\hline 125 & Higuerón & Ficus insipida Willd. & Moraceae & Látex & 0.25 \\
\hline 126 & $\begin{array}{l}\text { Hilo propio o } \\
\text { amor propio }\end{array}$ & Hedychium coronarium J.Koenig & Zingiberaceae & Medicinal-Ornamental & $0.08-0.23$ \\
\hline 127 & Hinojo & Foeniculum vulgare Mill. & Apiaceae & Medicinal & 0.02 \\
\hline 128 & Hobo & Spondias mombin L. & Anacardiaceae & Alimento & 0.19 \\
\hline 129 & Inbiana & Pavonia sp. & Malvaceae/Malvoideae & Medicinal & 0.04 \\
\hline 130 & Incibe & Aniba sp. & Lauraceae & Artesanal & 0.02 \\
\hline 131 & Insulina & Justicia chlorostachya Leonard. & Acanthaceae & Medicinal & 0.10 \\
\hline 132 & Iraca, bellota & Carludovica palmata Ruiz y Pavón & Cyclanthaceae & Alimento-Artesanal & 0.021 \\
\hline 133 & Jaboncillo & Isertia pittieri Standl. & Rubiaceae & Medicinal & 0.08 \\
\hline 134 & Jaguo & Genipa americana L. & Rubiaceae & Colorante & 0.68 \\
\hline 135 & Jigua negro & Ocotea cernua (Nees) Mez. & Lauraceae & Artesanal & 0.02 \\
\hline 136 & Jingurú & Ischnosiphon arouma (Aubl) Korn & Maranthaceae & Artesanal & 0.18 \\
\hline 137 & Lechero & Brosimum utile (Kunth) Pittier & Moraceae & Artesanal-Látex & $0.02-0.77$ \\
\hline 138 & Lecho o lechero & Tabernaemontana sp. & Apocynaceae & Alimento & 0.04 \\
\hline 139 & Limón & Citrus $x$ limon (L.) Burm.f. & Rutaceae & Medicinal & 0.02 \\
\hline 140 & Lirio & Couma macrocarpa Barb. Rodr. & Apocynaceae & Medicinal-Alimento-Látex & $0.06-0.25-0.54$ \\
\hline 141 & Llanten & Plantago major L. & Plantaginaceae & Medicinal & 0.30 \\
\hline 142 & Madroño & Garcinia madruno (Kunth) Hammel & Clusiaceae & Alimento-Combustible & $0.40-0.04$ \\
\hline \multirow[t]{2}{*}{143} & Manchará & Vismia macrophylla Kunth. & Hypericaceae & Medicinal-Colorante & $0.02-0.02$ \\
\hline & & & & Combustible-Látex & $0.04-0.35$ \\
\hline 144 & Mano de león & & & Ornamental & 0.12 \\
\hline 145 & Manteco & & Sapotaceae & Combustible-Látex & $0.02-0.02$ \\
\hline 146 & Marañón & Syzygium malaccense (L). Merr. y Perry & Myrtaceae & Alimento-Medicinal & 0.04-0.02 \\
\hline 147 & Mastranco & Salvia palaefolia H.B.K. & Lamiaceae & Medicinal & 0.02 \\
\hline 148 & Mata palo & Coussapoa villosa Poepp. y Endl. & Moraceae & Combustible-Látex & $0.02-0.06$ \\
\hline 149 & Mata ratón & Gliricidia sepium (Jacq.) Kunth & Fabaceae/Faboideae & Medicinal & 0.23 \\
\hline 150 & Matamba & Desmoncus cirrhifera A.H.Gentry y Zardini & Arecaceae & Alimento-Artesanal & $0.02-0.25$ \\
\hline 151 & Matapalo & Clusia sp. & Clusiaceae & Látex & 0.02 \\
\hline 152 & Menta & Menta piperita L. & Lamiaceae & Medicinal & 0.12 \\
\hline 153 & Michiraca & & & Alimento & 0.02 \\
\hline 154 & Miel quemada & Maripa panamensis Hemsl. & Convolvulaceae & Alimento & 0.06 \\
\hline 155 & Mil pesos & Oenocarpus bataua Mart. & Arecaceae & Alimento-Artesanal & $0.83-0.04$ \\
\hline 156 & Millonaria & Polyscias sp. & Araliaceae & Mágico religiosa-Ornamental & $0.04-0.02$ \\
\hline 157 & Moisés & Coix lacryma-jobi L. & Poaceae & Ornamental & 0.02 \\
\hline 158 & Monita, coquito & $\begin{array}{l}\text { Coccocypselum lanceolatum } \\
\text { (Ruiz y Pav.) Pers. }\end{array}$ & Rubiaceae & Medicinal & 0.10 \\
\hline 159 & Morita & Chlorophora tinctoria L. & Moraceae & Colorante & 0.02 \\
\hline 160 & Murrapo & Euterpe cuatrecasana Dugand & Arecaceae & Alimento-Artesanal & $0.10-0.10$ \\
\hline 161 & Nacedera & Trichanthera gigantea (Bonpl.) Nees & Acanthaceae & Medicinal-Combustible & $0.06-0.02$ \\
\hline 162 & Nispero & Chrysophyllum sp. & Sapotaceae & Combustible & 0.02 \\
\hline 163 & $\begin{array}{l}\text { Nuanamo/ } \\
\text { sangre de gallo }\end{array}$ & Virola sp. & Myristicaceae & Colorante-Látex & $0.02-0.10$ \\
\hline 164 & Oquendo & Maquira sp. & Moraceae & Combustible & 0.25 \\
\hline 165 & Oregano & Coleus amboinicus Lour & Verbenaceae & Medicinal & 0.04 \\
\hline 166 & Ortiga & Urtica sp. & Urticaceae & Medicinal & 0.02 \\
\hline 167 & Pacó de monte & Gustavia superva H.B.K & Lecythidaceae & Alimento & 0.27 \\
\hline 168 & Paco guitarro & Cespedesia macrophylla Seem. & Ochnaceae & Alimento-Combustible & $0.02-0.10$ \\
\hline 169 & Pacunda & Bidens pilosa L. & Asteraceae & Medicinal-Ornamental & 0.04-0.02 \\
\hline 170 & Paico & Chenopodium ambrosioides $\mathrm{L}$. & Chenopodiaceae & Medicinal & 0.27 \\
\hline 171 & Palma de cristo & Cordyline rubra Kunth & Agavaceae & Ornamental-Mágico religiosa & $0.38-0.02$ \\
\hline 172 & Palo de ají & & & Tóxica & 0.06 \\
\hline 173 & Palo perico & Simarouba amara Aubl. & Simaroubaceae & Combustible & 0.33 \\
\hline 174 & Panocre & & Orchidaceae & Mágico religiosa & 0.02 \\
\hline 175 & Papaya & Carica papaya L. & Caricaceae & Mágico religiosa-Látex & $0.02-0.04$ \\
\hline 176 & $\begin{array}{l}\text { Papayuela de } \\
\text { monte }\end{array}$ & Dracontium spruceanum (Schott) G. Zhu & Araceae & Medicinal & 0.08 \\
\hline 177 & Pega paruma & & & Mágico religiosa & 0.02 \\
\hline 178 & Pichindé & Pithecellobium longifolium Standl. & Fabaceae/Mimosoideae & Combustible & 0.15 \\
\hline 179 & Pichindecillo & Calliandra glaberrima (Benth.) Britton y Killip & Fabaceae/Mimosoideae & Ornamental & 0.02 \\
\hline 180 & Pinguací & Jacaranda hesperia Dugand. & Bignoniaceae & Medicinal-Combustible & $0.12-0.35$ \\
\hline 181 & Piña de monte & Ananas sp. & Bromeliaceae & Alimento & 0.02 \\
\hline 182 & Piña de monte & Guzmania eduardi Andre ex Mez. & Bromeliaceae & Ornamental & 0.04 \\
\hline
\end{tabular}


Anexo 1. Lista de las especies de PFNM utilizadas en el corregimiento Doña Josefa, Chocó, Colombia, con sus respectivos nombres vulgares y valores de uso por categoría (continuación)

\begin{tabular}{|c|c|c|c|c|c|}
\hline \multirow[t]{2}{*}{ No } & \multicolumn{2}{|c|}{ Nombre } & \multirow[t]{2}{*}{ Familia } & \multirow[t]{2}{*}{ Usos } & \multirow[t]{2}{*}{ IVU } \\
\hline & vulgar & científico & & & \\
\hline 183 & Pita & $\begin{array}{l}\text { Aechmea magdalenae (André) } \\
\text { André ex Baker }\end{array}$ & Bromeliaceae & Artesanal & 0.02 \\
\hline 185 & Platanillo & Heliconia sp. 1 & Heliconiaceae & Ornamental & 0.46 \\
\hline 186 & Platanillo & Heliconia sp. 2 & Heliconiaceae & Artesanal & 0.21 \\
\hline 187 & Poleo & Satureia brownei Sw. (Briq.) & Lamiaceae & Medicinal & 0.02 \\
\hline 188 & Potré & Thoracocarpus bissectus (Vell.). Harling. & Cyclanthaceae & Artesanal & 0.52 \\
\hline 189 & Pringamosa & Urera baccifera (L.) Gaudich. ex Wedd & Urticaceae & Medicinal-Tóxica & $0.10-0.04$ \\
\hline 190 & Pronto alivio & Origanum maioranna & Lamiaceae & Medicinal & 0.06 \\
\hline 191 & Quereme & Peperomia sp. & Piperaceae & Mágico religiosa & 0.41 \\
\hline 192 & $\begin{array}{l}\text { Raya sardinal } \\
\text { rabo sardina }\end{array}$ & Columnea picta H. Karst & Gesneriaceae & Medicinal & 0.08 \\
\hline 193 & Resucito & Hibiscus rosea L. & Malvaceae/Malvoideae & Medicinal-Ornamental & $0.02-0.21$ \\
\hline 194 & Riñonera & Columnea consanguinea Hanst. & Gesneriaceae & Medicinal & 0.52 \\
\hline 195 & Ruda castilla & Ruta graveolens L. & Rutaceae & Medicinal-Mágico religiosa & $0.10-0.57$ \\
\hline 196 & Sábila & Aloe sp. & Asphodelaceae & Medicinal-Mágico religiosa & $0.02-0.10$ \\
\hline 197 & Salvia & & Lamiaceae & Tóxica & 0.02 \\
\hline 198 & Santa María boba & Piper peltatum L & Piperaceae & Medicinal & 0.27 \\
\hline 199 & Santa María de anís & Piper auritum Kunth. & Piperaceae & Medicinal & 0.14 \\
\hline 200 & Sauco & Solanum sp. & Solanaceae & Medicinal & 0.02 \\
\hline 201 & Sauco castilla & Sambucus nigra L. & Caprifoliaceae & Medicinal & 0.29 \\
\hline 202 & Secá/amonseco & Erythrina edulis Triana & Fabaceae/Faboideae & Medicinal & 0.04 \\
\hline 203 & Siempre viva blanca & Tripogandra serrulata (Vahl) Handlos & Commenilaceae & Medicinal & 0.06 \\
\hline 204 & Siempre viva morada & Tradescantia cumanensis Kunth. & Commenilaceae & Medicinal & 0.08 \\
\hline 205 & Sombrerito del diablo & Psychotria poeppigiana Müll. Arg. & Rubiaceae & Medicinal-Ornamental & $0.08-0.02$ \\
\hline 206 & Suelda consuelda & Elephantopus mollis Kunth & Asteraceae & Medicinal & 0.12 \\
\hline 207 & Tachuelo & Zanthoxylum sp. & Rutaceae & Combustible & 0.02 \\
\hline 208 & Tapa cula & Pentagonia microphylla Benth. & Rubiaceae & Alimento & 0.23 \\
\hline 209 & Taparo & Sloanea sp. & Elaeocarpaceae & Combustible & 0.04 \\
\hline 210 & Taparo & Attalea allenii H.E. Moore & Arecaceae & $\begin{array}{l}\text { Alimento-Artesanal } \\
\text { Mágico religiosa }\end{array}$ & $\begin{array}{r}0.87-0.08 \\
0.14\end{array}$ \\
\hline 211 & Tasi (helecho) & Alsophila tarapotensis Rosenst. & Cyatheaceae & Medicinal & 0.18 \\
\hline 212 & Te & & Lamiaceae & Medicinal & 0.02 \\
\hline 213 & Toronjil & Melissa officinalis L. & Lamiaceae & Medicinal & 0.06 \\
\hline 214 & Tortugo & Cydista aequinoctialis (L.) Miers & Bignoniaceae & Artesanal & 0.06 \\
\hline 215 & Tres dedos o costeña & Piper tricuspe (Miq. ) C. Dc. & Piperaceae & Medicinal & 0.18 \\
\hline 216 & Varejón & Vernonia sp. & Asteraceae & Tóxica & 0.02 \\
\hline 217 & Venadillo & Neurolaena lobata (L.) Cass. & Asteraceae & Medicinal & 0.08 \\
\hline 218 & Venturosa & Lantana urticifolia P. Mill. & Verbenaceae & Medicinal & 002 \\
\hline 219 & Verdolaga & Portulaca oleracea L. & Portulacaceae & Medicinal & 0.04 \\
\hline 220 & Zapote de monte & Matisia cordata Bonpl. & Malvaceae/Bombacoideae & Alimento & 0.17 \\
\hline 221 & Zapotillo & & Sapotaceae & Alimento & 0.04 \\
\hline
\end{tabular}

